\title{
44519 - COMPLIANCE TO STANDARD BODY SUBSTANCE PRECAUTIONS BY OPERATING ROOM PERSONNEL
}

\author{
Isabella Devito, Mount Sinai Hospital, Toronto, ON, Canada; \\ Cheryl Simpson, University of Toronto; \\ Sharon Davies, Mount Sinai Hospital;
}

INTRODUCTION: Operating room (OR) personnel are at high risk of contact with body fluids and in turn of acquiring or transmitting disease. Therefore standard body substance precautions (BSPs) should be utilized at all times. While OR personnel are aware that BSPs should be utilized, they may not be totally compliant. No study to date, has investigated compliance to BSPs in the OR in a prospective observational manner, nor investigated obstacles to compliance. The purpose of this study was to determine the incidence of compliance to standard BSPs and obstacles to compliance in a tertiary care OR.

METHODS: Two checklists were developed. The first was designed to document compliance of OR personnel with standard BSPs; the second to document the availability of the necessary equipment. After approval by the local Human Research Ethics Board, consent was obtained from OR personnel. A trained research assistant observed intravenous cannulation and airway management. Descriptive statistics focused on compliance with intavenous insertion, intubation and availability of necessary equipment.

RESULTS: A total of 117 observations were completed for intravenous cannulation. The compliance to wearing gloves was $95 \%$ for the anesthesiologist and $72 \%$ for the assistant. The intravenous cannula was handled safely post insertion $89 \%$ of the time. The local anesthetic needle was handled safely $20 \%$ of the time. A total of 128 observations were completed for intubation. Compliance to wearing gloves was $99 \%$ for the anesthesiologist and $85 \%$ for the assistant. Anesthesiologists removed their gloves 50\% of the time and the assistant $67 \%$ following intubation. A total of 135 observations were completed for documentation of availability of the necessary equipment for standard BSPs. Gloves in varying sizes, hand antiseptic and disinfectant for equipment were available $96 \%, 67 \%$ and $59 \%$ of the time respectively. The hazardous waste container was full on $7 \%$ of occasions. A container for disposal of used airway equipment was available only $64 \%$ of the time.

DISCUSSION: Compliance to wearing gloves for both intravenous cannulation and intubation is high for anesthesiologists. The compliance for the assistant was lower. The appropriate handling of local anesthetic needles post intravenous insertion is extremely low. This finding indicates the need for more education regarding the safe disposal of needles or alternate techniques for their disposal. Following intubation, 50\% of anesthesiologists removed their gloves. As a result, there could be contamination of the anesthetic machine leading to potential cross contamination between patients. Educational programs should be initiated to deal with this. As well, OR managers need to be made aware of the lack of supplies necessary for complete compliance. These issues are extremely important in light of our past experience with SARS and our potential exposure to new pathogens. 